\title{
Gamma-H2AX Foci Counting: Image processing and control software for high-content screening
}

\author{
P. R. Barber", R.J. Locke, G. P. Pierce, K. Rothkamm and B. Vojnovic \\ University of Oxford Gray Cancer Institute, Mount Vernon Hospital, Northwood, HA6 2JR, UK.
}

\begin{abstract}
Phosphorylation of the chromatin protein $\mathrm{H} 2 \mathrm{AX}$ (forming $\gamma \mathrm{H} 2 \mathrm{AX}$ ) is implicated in the repair of DNA double strand breaks (DSB's); a large number of H2AX molecules become phosphorylated at the sites of DSB's. Fluorescent staining of the cell nuclei for $\gamma \mathrm{H} 2 \mathrm{AX}$, via an antibody, visualises the formation of these foci, allowing the quantification of DNA DSB's and forming the basis for a sensitive biological dosimeter of ionising radiation.

We describe an automated fluorescence microscopy system, including automated image processing, to count $\gamma \mathrm{H} 2 \mathrm{AX}$ foci. The image processing is performed by a Hough transform based algorithm, CHARM, which has wide applicability for the detection and analysis of cells and cell colonies. This algorithm and its applications for cell nucleus and foci detection will be described. The system also relies heavily on robust control software, written using multi-threaded cbased modules in LabWindows/CVI that adapt to the timing requirements of a particular experiment for optimised slide/plate scanning and mosaicing, making use of modern multi-core processors. The system forms the basis of a general purpose high-content screening platform with wide ranging applications in live and fixed cell imaging and tissue micro arrays, that in future, can incorporate spectrally and time-resolved information.
\end{abstract}

Keywords: Gamma-H2AX, High-content screening, Image processing, Hough transform, dosimetry

\section{INTRODUCTION}

The study of damage to cellular DNA is essential for the understanding of cell apoptosis and mutation that may lead to serious disease including cancer. Changes in the genome caused by a single double strand break (DSB) may be enough to induce cancer or cell death ${ }^{1}$. A mechanism by which DSBs can be located within the nucleus and quantified is highly desirable. We can make use of the cell's natural protein function targeted at the repair of such breaks to reveal break locations under fluorescence microscopy.

Phosphorylation of the chromatin protein $\mathrm{H} 2 \mathrm{AX}$ (forming $\gamma \mathrm{H} 2 \mathrm{AX}$ ) is implicated in the DSB repair pathway; a large number of H2AX molecules become phosphorylated at the sites of DSB's. Fluorescent staining of the cell nuclei for $\gamma \mathrm{H} 2 \mathrm{AX}$, via an antibody, enables us to visualise the formation of these foci, allowing the quantification of DNA DSB's and forming the basis for a sensitive biological dosimeter of ionising radiation. There is a one to one correspondence between the number of DSB's and $\gamma \mathrm{H} 2 \mathrm{AX}$ foci ${ }^{2}$.

Counting and quantifying the $\gamma \mathrm{H} 2 \mathrm{AX}$ foci necessarily requires probing individual, intact cells. Because of the probabilistic nature of DSBs occurring due to a given damaging insult, the systematic study of the formation of $\gamma \mathrm{H} 2 \mathrm{AX}$ foci in response to DNA damaging insults requires large numbers of experiments involving large sample numbers for statistical robustness. The foci of hundreds of cells may have to be counted. This quickly becomes very time consuming and the automation of systems to image cells and record and analyse data becomes a worthwhile pursuit.

In common with other experiments, average absorption or emission type measurements, where counting is not required, may be good enough in some instances but in any case have to be validated against counting. The variation in foci fluorescence intensity and background staining usually dictates that these methods are insufficient and that the counting

\footnotetext{
*barber@gci.ac.uk; phone +44 1923 828-611; fax +44 1923 835-210; www.gci.ac.uk
} 
of individual foci is the most reliable indicator, which is easily comparable between experiments and centres. An automated method provides robustness through objectivity and can work for consistently for longer hours.

We describe such a system, targeted at the problem of counting $\gamma \mathrm{H} 2 \mathrm{AX}$ foci. The measurement of additional parameters is also possible with the image processing algorithms employed. Such additional information such as foci diameter, intensity and position, may lead to further studies, for example see Hamada et al. ${ }^{3}$

Many of the hardware and software techniques employed are modular and have been used to form other systems for other assays and experiments that require the investigation of large numbers of cells. We employ a modular software approach and novel image processing based on a compact Hough transform ${ }^{4}$. Multithreading of processes on the host computer has lead to a system capable of efficiently performing large numbers of repetitive actions but is adaptable to the actual sequence and timing of events chosen at run time. The systems thus may form the basis for high-content screening for cells in vitro and tissue micro arrays. We are developing time-resolved techniques that will offer fluorescence lifetime measurement under this or similar automated platforms.

\section{METHODS}

\subsection{Hardware and Control Software}

The microscopy platform is based around an upright motorised Nikon Eclipse 90i system (Nikon, UK), fitted with an epi-fluorescence attachment and image zoom optics (Fig. 1). Fluorescence detection is performed using standard Hg arc lamp excitation, in conjunction with conventional dichroic filter cubes (UV-2E/C "DAPI", B-2E/C "FITC" and G-2E/C "TRITC"). The microscope was fitted with two Nikon CFI Plan Fluor air objective lenses: 10X 0.30 NA and 40X 0.75 NA. Z axis (focus) control is performed using the 90i's focus drive and control of the microscope is through the Nikon 90i SDK for Windows (version 1.0.63.73) and the Nikon USB Eclipse-i Microscope driver which is part of the iSeries Tools (version 1.50). The microscope is fitted with a Märzhäuser 'SCAN' motorised stage (100 x $100 \mathrm{~mm})$ with a custom insert to hold four slides, controlled by a 'Corvus 2-axis' XY controller (Märzhäuser Wetzlar GmbH, Germany) that allows fast sample positioning to a resolution of $\sim 125 \mathrm{~nm}$. Image acquisition is performed with a cooled Hamamatsu ORCA-ER camera, operating at a maximum resolution of 1344 x 1024 pixels, with image transfer at 10 frames $\mathrm{sec}^{-1}$ typically, via a 'Firewire' interface. All control and acquisition software tools have been developed inhouse, based on re-usable software modules developed for other similar purposes. Development was in the LabWindows/CVI environment (National Instruments, UK). A PC based on an Intel Pentium D830 dual core (2 x 3.0 $\mathrm{GHz}$ ) processor with $1 \mathrm{~Gb}$ RAM is used in conjunction with two SXGA graphics screens to control the system.

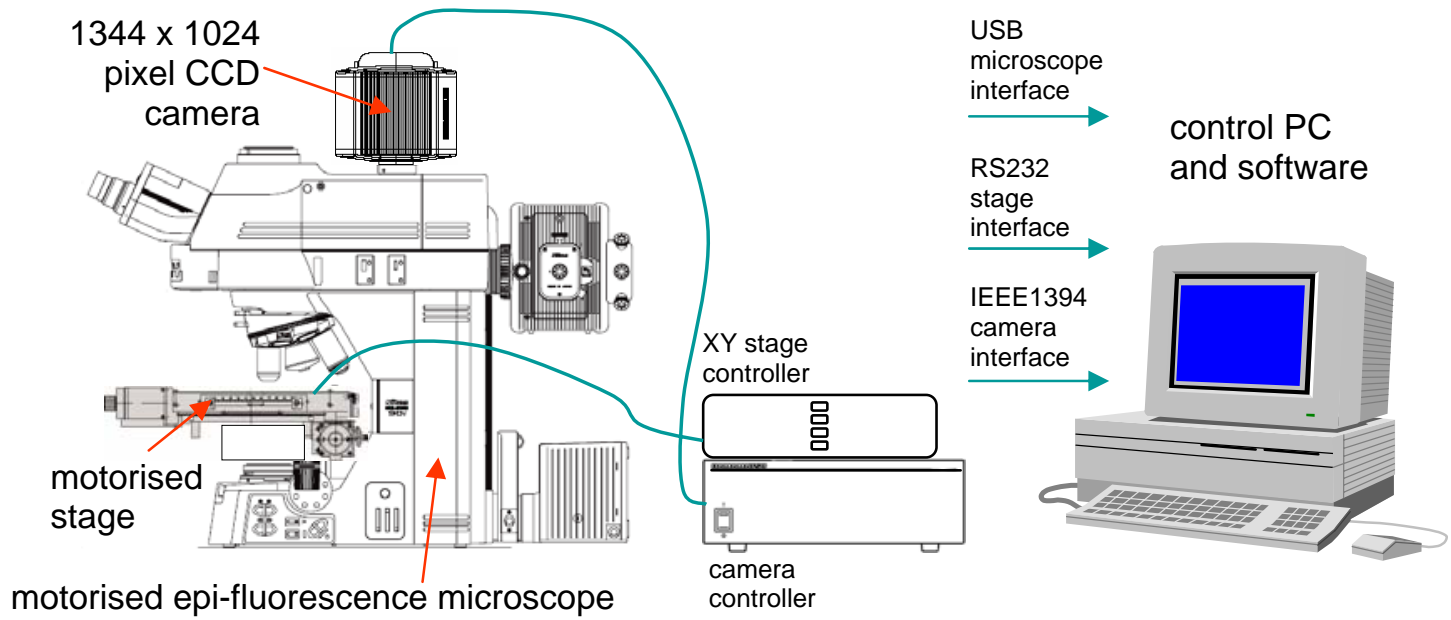

Fig. 1. Schematic representation of the major components of the system. 
Multithreading and message passing is used throughout the program. Multithreading allows different hardware elements to be controlled and active simultaneously where permitted, and allows the system to adjust to different hardware timing requirements for different experiments. For example, after the capture of an image, image transfer from the camera to the PC and stage motion to bring the next cell into the field of view are allowed to proceed simultaneously. As it is not known which of these two procedures will take the longest time, for any given experiment, message passing allows threads to report when operations are complete to enable on-the-fly adaptation to different experiments.

\subsection{Sample Preparation}

Cells were grown in Eagle's Minimum Essential Medium (Cambrex, Nottingham, UK) supplemented with $10 \%$ foetal calf serum and antibiotics at $37^{\circ} \mathrm{C}$ in a humidified atmosphere containing $5 \% \mathrm{CO}_{2}$. They were $\mathrm{X}$-irradiated at room temperature as stationary monolayers on coverslips and then incubated for various times at $37^{\circ} \mathrm{C}$.

Cells were either fixed in 3.7\% paraformaldehyde in PBS (15 min at room temperature) and permeablised using $0.5 \%$ Triton X 100 in PBS $\left(5 \mathrm{~min}\right.$ at $\left.4^{\circ} \mathrm{C}\right)$ or fixed in $100 \%$ methanol $\left(10 \mathrm{~min}\right.$ at $\left.-20^{\circ} \mathrm{C}\right)$. Samples were blocked in phosphatebuffered saline (PBS) with $2 \%$ bovine serum albumin (BSA) for 3 × $5 \mathrm{~min}$ at room temperature. Samples were incubated with anti- $\gamma \mathrm{H} 2 \mathrm{AX}$ antibody (clone JBW301, Upstate, Charlottesville, VA) overnight at $4^{\circ} \mathrm{C}$, washed in PBS, $2 \%$ BSA for $2 \times 5 \mathrm{~min}$, and incubated with Alexa Fluor 488-conjugated goat anti-mouse secondary antibody (Invitrogen, Paisley, UK) for $1 \mathrm{~h}$ at room temperature. Coverslips were washed in PBS for $2 \times 2$ min, stained with 4,6 diamidino-2-phenylindole (DAPI) for $5 \mathrm{~min}$, washed for $2 \mathrm{~min}$ in PBS and mounted onto slides using Mowiol Mounting Medium.

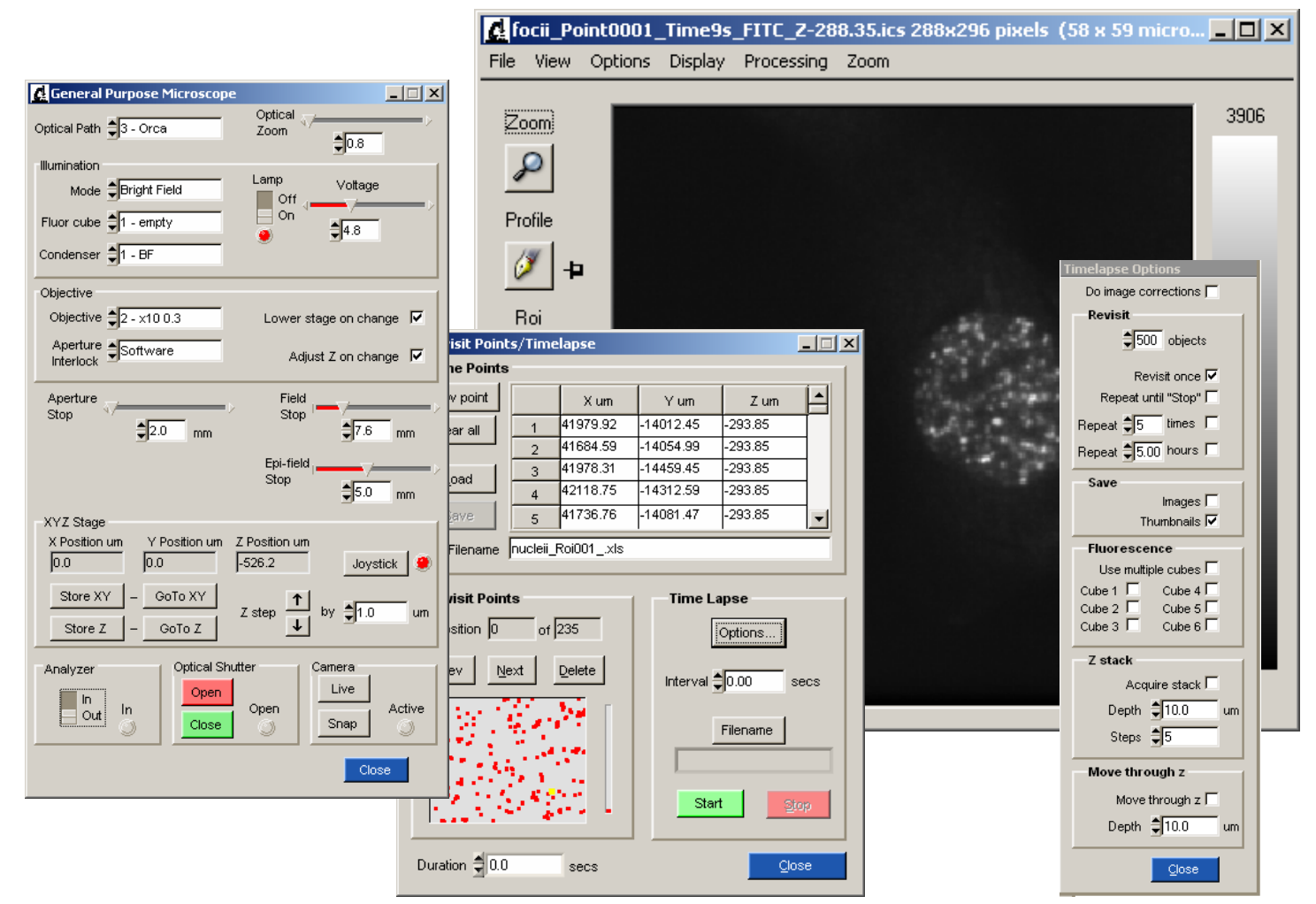

Fig. 2. Screenshots of the control and acquisition software showing general microscope control, cell mapping and revisiting together with the main image window. 


\subsection{Image Acquisition}

The acquisition software permits a range of acquisition modes, over space (image tiling) and time (time-lapse) and provides capabilities for 'revisiting' coordinates, see Fig. 2. A particular subset of these general functions is used for this application.

Two procedures are generally used in the course of an experiment. It is usual that the cells are sparse such that generally not more that one cell is imaged within the field of view at the resolution required to identify foci (using the 40X objective). In this case a two pass approach is used, where during the first "object finding" stage, cells are found and mapped. During this stage the 10X objective was selected along with the DAPI fluorescence filter cube. The Compact Hough And Radial Map (CHARM) algorithm (see section 2.4) was used during this stage to segment cell nuclei such that their centres can be mapped. The ability of this algorithm to separate touching objects can be used to flag the presence of cell clumps or doublets. An automated procedure to define a focus plane has been implemented which requires the user to focus, by eye, at 3 points on the slide. Information on the position, size and intensity for each object is recorded such that a map of cells suitable for re-visiting is produced. The stage is moved such that the whole slide area is assessed by tiling images together in a meander pattern with a small overlap between images.

The second acquisition stage involves revisiting the mapped cell nuclei, at high-optical-power to record images for detailed analysis. The 40X objective is selected and images are acquired centred on each mapped nucleus with both the DAPI and FITC fluorescence cubes. It is often necessary to set a separate camera gain and/or exposure for each cube as the recorded fluorescence intensity may differ greatly between channels, and this is possible with our software. During this stage it is only necessary to acquire of sub-array of the available pixels around the nucleus near the centre of the field of view. This impacts on the time required for acquisition and analysis, and image data storage, as well as allowing the epi-field stop diameter to be reduced to minimise the exposure of other nuclei to UV excitation light. At this stage it is also possible to acquire a random selection of nuclei if there is a desire to reduce acquisition time at the expense of statistical accuracy.

Two image acquisition methods are being evaluated for this second acquisition stage, aiming to capture an in-focus image of all foci, distributed in 3 dimensions, in a single 2-dimensional image (see Fig. 3). The first is the more traditional 'through focus stack' followed by a maximum intensity projection. The second is an 'extended focus' image produced by changing the focal plane of the microscope simultaneously with the acquisition of a single frame by the camera. This is faster to acquire and produces visually better results. The focal plane should traverse the cell nucleus during the acquisition. The raw image acquired contains a significant amount of out-of-focus information but this can be largely removed through deconvolution as the motion of the focal plane can be determined. This degree of complication is not required, however, as excellent results can be achieved with the 'unsharp mask' filter $^{5}$ in place of true deconvolution.

\subsection{Foci analysis}

Foci analysis is performed off-line in a separate program called "TRI2" (version 2.2) which has been developed over a number of years for different purposes. It includes batch processing functions and automatically sorts the images acquired at the microscope according to cell number and cube type, applies image analysis and outputs the results directly into a Microsoft Excel spreadsheet.

The algorithm used for foci counting and analysis is the Compact Hough and Radial Map (CHARM) method ${ }^{4}$ aimed at faint and ill-defined shapes. It uses edge information and a compact Hough transform to highlight the centres of circular objects (see Fig. 4). Two perpendicular Sobel operators are used to detect edge pixels and derive directional information; these are thresholded to find significant 'edgels' which are used to increment values in an accumulator space along a radius of values towards the light side of the edge, between the assumed minimum and maximum object radii. Peaks in the resultant accumulator space mark the centres of circular objects since many edgels may contribute to a common centre. Radial Maps based on these detected centres are formed by searching outwards to border edge points. Overlapping objects are separated or merged according to shape-processing criteria. 

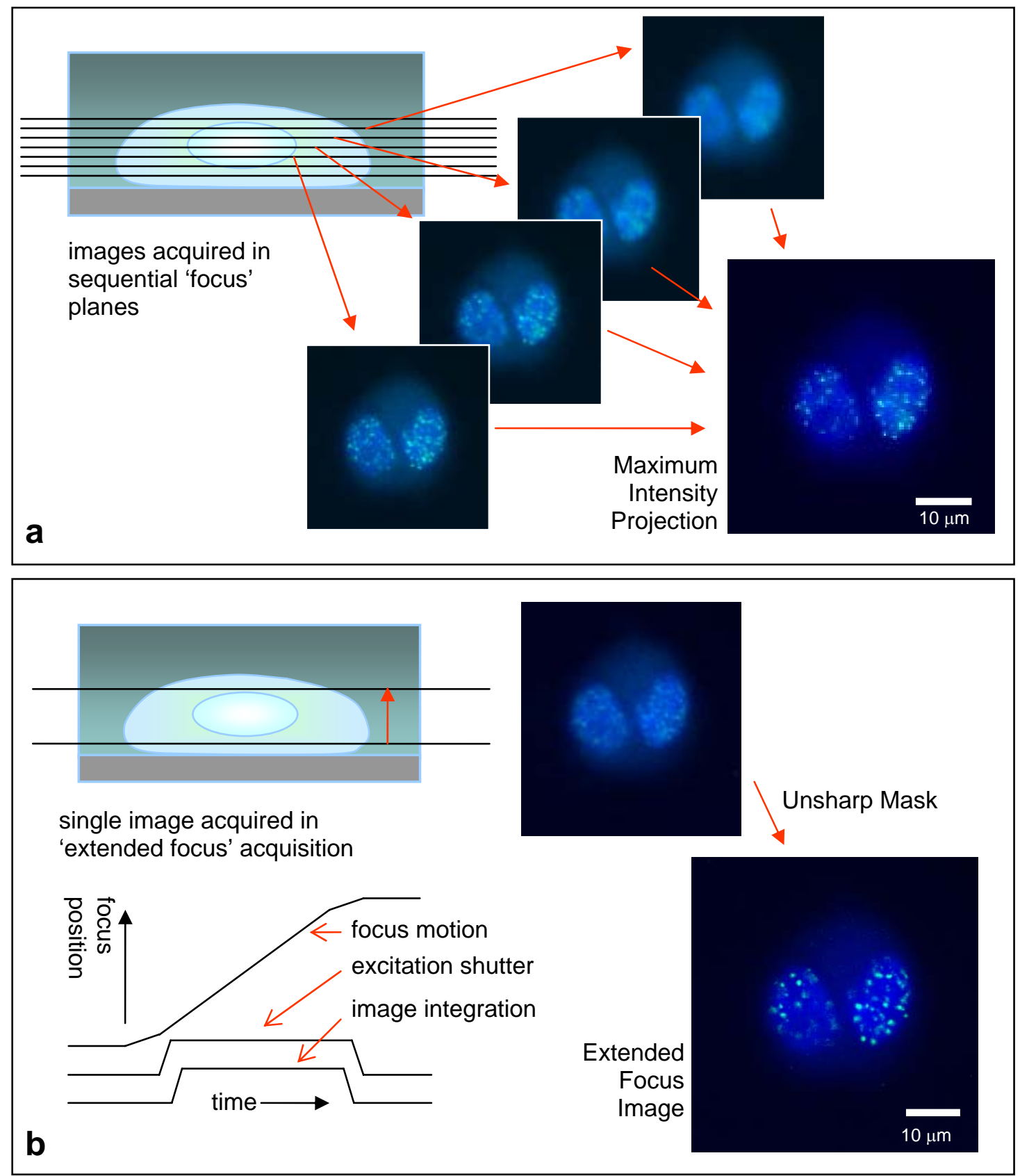

Fig. 3. Two acquisition modes are under evaluation to capture the foci, distributed in 3D, in a 2D image. Subimage 'a' shows a traditional 'through focus stack' and their combination in a 'maximum intensity projection'. Sub-image ' $b$ ' shows how an 'extended focus image' may be obtained by changing the focal plane simultaneously with the acquisition of a single frame and then applying deconvolution-like image processing such as the 'unsharp mask'.

The CHARM algorithm has several steps and to aid the user in selecting suitable parameters for each step an "optimizer" has been written. This allows the user to step through the algorithm, adjust the parameters relevant to each stage and quickly see the outcome at each stage. Parameter sets can also be stored for future use. CHARM is a very flexible algorithm but as such does require some setting up and some prior knowledge about the approximate size of the object of interest. 
With different parameters, the same algorithm is used to detect nuclei and foci separately from images of DAPI and Alexa-488 fluorescence respectively. The radial maps are then used to evaluate additional statistics such as foci position, diameter, intensity, shape factor and distance to its nearest neighbour. The batch process outputs all this information in tabulated form into a text file or Microsoft Excel.

\section{RESULTS}

\subsection{Image Acquisition}

Both acquisition procedures; coarse nuclei finding and high-power image acquisition, have been automated to require no user intervention and in a time-efficient manner. However, the acquisition speed is severely limited in this application by the time taken by the Nikon microscope driver to execute a command and return to the calling program. This introduces delays of several hundred milliseconds to each call. In other systems developed within this group ${ }^{6,7}$, the time required to open or close an optical shutter or for short focus changes is typically a few 10's of milliseconds. Use of the Nikon software and hardware for these functions introduces unacceptable delays. If this delay is not removed in future releases of the Nikon drivers, the only option for future development is to introduce third-party hardware and drivers with faster response times as in our previous systems.

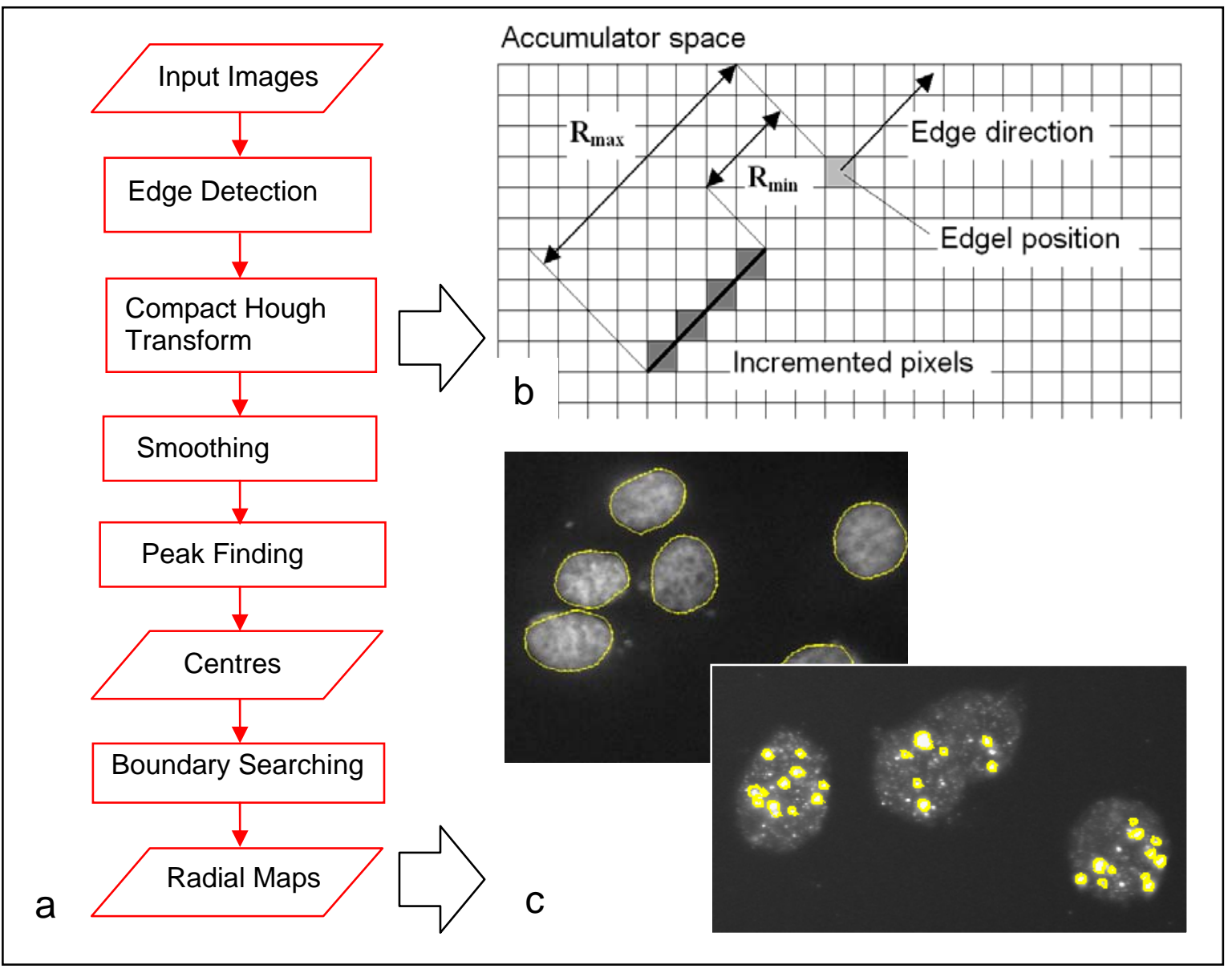

Fig. 4. Flow chart showing the main elements of the Compact Hough And Radial Map (CHARM) algorithm (a). The accumulator space of the Hough transform is populated with lines emanating from edge pixels, given the expected minimum and maximum object radius (b). The radial maps produced outline the nuclear or foci objects (c). 
With the limitations of the Nikon drivers in mind, the time taken for cell/nuclei finding is approximately 30 seconds for a scan covering $6 \mathrm{~mm}^{2}$ containing approximately 350 cells.

Revisiting the cells to obtain high-power images in the second acquisition stage was generally performed using all 3 epifluorescence cubes, for completeness. Two acquisition sequences are therefore possible: to move to each cell position and change cubes in place; or to move to every position with one cube, then with the next cube and so on. The first method may have positioning advantages as repeatedly revisiting all the cells may lead to cumulative stage errors in a stage with open-loop control, the second may have speed advantages. The two image acquisition methods described in section 2.3 were also used, leading to a further differentiation in acquisition times which are detailed in Table 1.

\subsection{Image Analysis}

The speed of analysis of the foci images, performed offline in TRI2, is dependant on the host PC but is typically 5 seconds per cell on today's desktop computers. The batch processing function can be run on a large number of images and includes the application of the maximum intensity projection or the unsharp mask as required together with two passes of the CHARM algorithm for nuclei and foci. Typical output from the image analysis stage is shown in Fig. 5. The CHARM algorithm is adaptable to many biological situations, and several different cell types have been tested, including MCF7, MOLT4, UT14, V79, 48BRhtert and Lymphocytes.

\subsection{Foci Analysis}

Many statistics are calculated on the nuclei and foci and are automatically stored in a spreadsheet. This detailed information, such as focus diameter, intensity, shape factor and position, is very difficult or near impossible to collect when manual counting.

An initial validation experiment has been performed to look at the time course of foci formation and disappearance. 48BRhtert cells were exposed to a $1 \mathrm{~Gy}$ dose of X-ray radiation and then incubated for a period of time before fixation and treatment to reveal the foci. Approximately 50 to 60 cells were then scored for foci number per cell at each time point both manually by eye by a single biologist (using images from the automated system) and using the automated algorithm. Through focus Z-stacks comprising 3 focal positions were used approximately $0.75 \mu \mathrm{m}$ apart. Fig. 6 shows the correlation between automated and manual counting in terms of foci per cell.

Table 1. Typical times for the differing sequencing of multiple cubes and image acquisition methods. Through focus stacks comprised 7 images of $100 \mathrm{~ms}$ exposure covering a focus distance of $3 \mu \mathrm{m}$. Extended focus images comprised a single 100-ms exposure image, also over $3 \mu \mathrm{m}$.

\begin{tabular}{|c|c|c|}
\hline Time per cell (seconds) & Through focus stack & Extended focus image \\
\hline Change FL cube at each cell & 20.2 & 10.5 \\
\hline Revisit all cells with each FL cube & 17.1 & 7.5 \\
\hline
\end{tabular}

\section{CONCLUSION}

A fully automated fluorescence microscope forms the basis for a high-content screening platform and a system aimed at the analysis of $\gamma \mathrm{H} 2 \mathrm{AX}$ foci has been described. The general purpose acquisition software aids experiment design, but in-house development allows easy optimisation and adaptation for specific tasks as well as time and cost-efficient development of working systems. The flexibility and speed of the CHARM algorithm offers a realistic route for automated processing for many biological situations imaged by fluorescence and brightfield microscopy. Automated processing of this type offers objective counting and the collection of additional statistics on size, shape, position and distance to the nearest neighbour.

System speed is currently similar to manual counting but future development will result in significant speed increase. This is currently limited by the Nikon SDK and driver for the 90i microscope and ten-fold increase should be possible over the times given in section 3.3. 
The system performance has been validated against a single manual foci count in an initial experiment to plot the time course of foci number per cell following irradiation. Example images are shown in Fig. 5 and the average foci per cell counts are shown in Fig. 6. The results for the automated counter are good but show slight under counting when foci numbers are high and over counting when numbers are low. Future validation must include additional manual counts to asses whether the system falls significantly outside the range of manual counting error and subjectivity, as has been found in previous studies (e.g. Lumley et al. ${ }^{8}$ ).

The obtained result that $\sim 30 \gamma \mathrm{H} 2 \mathrm{AX}$ foci were present in human $48 \mathrm{BRhtert}$ fibroblasts $0.5 \mathrm{~h}$ after exposure to $1 \mathrm{~Gy} \mathrm{X}$ rays was similar to the yield of DSBs that is induced per Gy of X-rays in a human cell, ${ }^{9,10}$. This indicates a one-to-one correlation between the number of X-ray-induced $\gamma \mathrm{H} 2 \mathrm{AX}$ foci and DSBs. The time course for the disappearance of foci after exposure to $1 \mathrm{~Gy}$ X-rays resembled the kinetics of DSB repair after 20 Gy X-rays, as measured by pulsed-field gel electrophoresis (PFGE) and was similar to kinetics reported for other primary human fibroblasts ${ }^{9,10}$. This finding provides further evidence that radiation-induced $\gamma \mathrm{H} 2 \mathrm{AX}$ foci represent DSBs. The slight temporal delay of foci loss compared to DSB repair in the first hour after irradiation suggests that the dephosphorylation of $\gamma-\mathrm{H} 2 \mathrm{AX}$ at the site of a DSB occurs about 30 min after the physical sealing of the break.

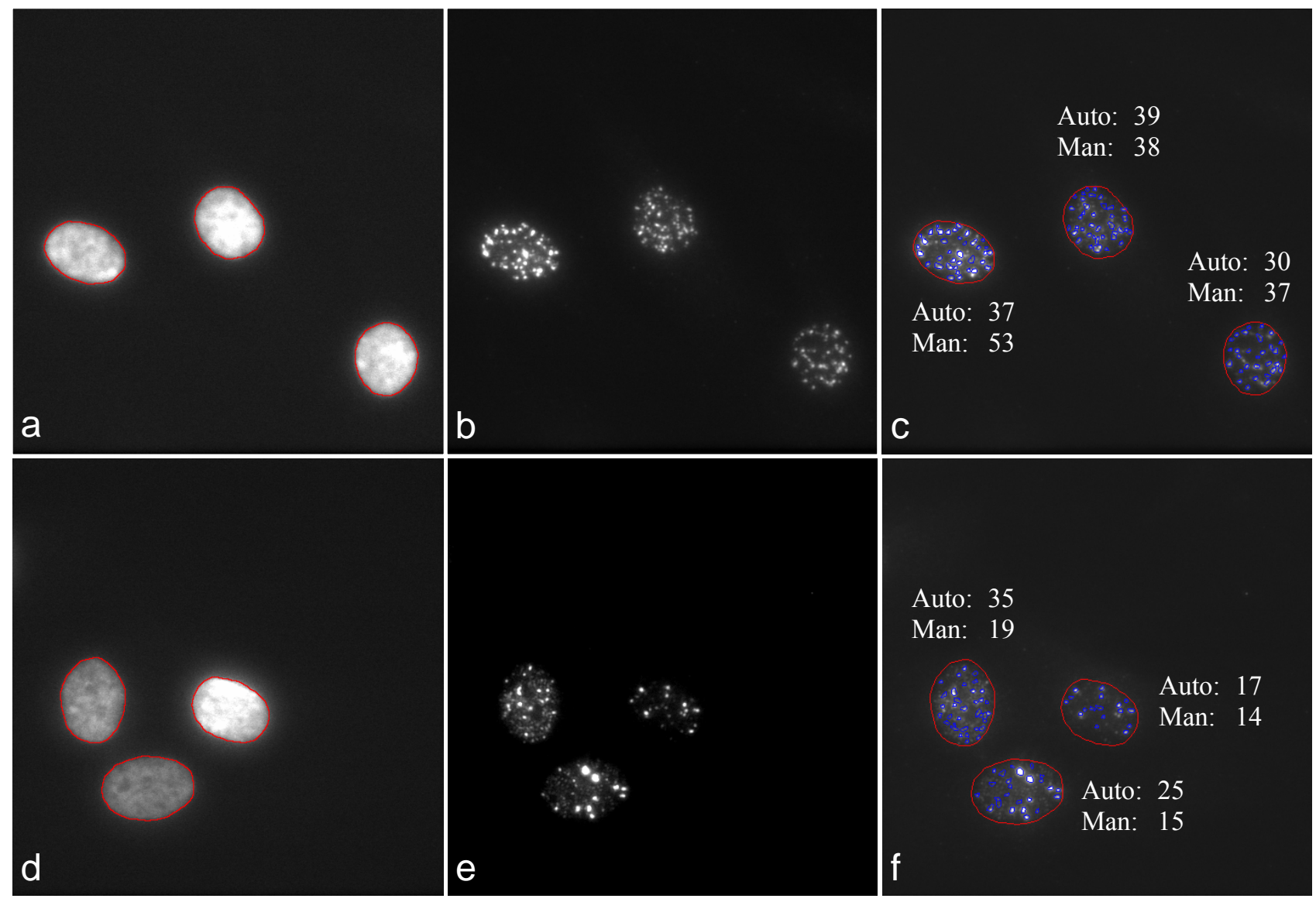

Fig. 5. Automated processing proceeds with the delineation of the cell nucleus from the image of DAPI fluorescence (a and d, detected outlines in red). Images of the Alexa-488 fluorescence show the foci locations (b and e). Counts of foci per nucleus are produced automatically ( $c$ and f) by combining the information from both DAPI and Alexa-488 images. The automated counts are shown with the equivalent manual count, performed by eye. The images are from a time course experiment with 48BRhtert cells, after irradiation with 1 Gy X-ray dose. Images $a, b$ and $c$ are after an incubation period of 30 minutes, near the peak of foci abundance, and $d$, e and $f$ are from the 6 hour time point where the foci number is decreasing.

Future work will involve an enhancement in system speed through the use of third-party hardware components, such as focus drive and fast optical shutter, to overcome the current limitations of the Nikon $90 \mathrm{i}$ as in our previous systems, or 
through the use of updated drivers with enhanced performance (which is obviously preferable). We are in discussion with Nikon UK and if some fundamental problem precludes the release of more efficient drivers then we shall be forced to re-consider other microscope manufacturers in future systems.

Data storage and easy retrieval is becoming more important as systems such as this come online. Future work is also ongoing to link this system with an image and metadata data-basing and processing environment such as the Open Microscopy Environment (OME) $)^{11,12}$. Such an environment will allow the automatic uploading of images and metadata to the central database at acquisition time and their retrieval, searching and analysis either immediately or offline from another location. It is only by thorough databasing that the results from these types of experiments can be linked and correlated with other data obtained in more classical genomics or proteomics experiments.

With a wider perspective, we are building systems aimed at other assays. In particular a system for high-throughput fluorescence lifetime imaging (FLIM) is being developed, which may utilise many of the hardware and software components described here together with a novel custom detection system, and will be the subject of future publications.

Many future experiments are planned, from a biology perspective, to help to elucidate the mechanism of formation of $\gamma \mathrm{H} 2 \mathrm{AX}$ foci and its dynamics. These will include the study of many of the new parameters that can now be collected, e.g. foci diameter, intensity and degree of clustering (obtainable from foci position information).

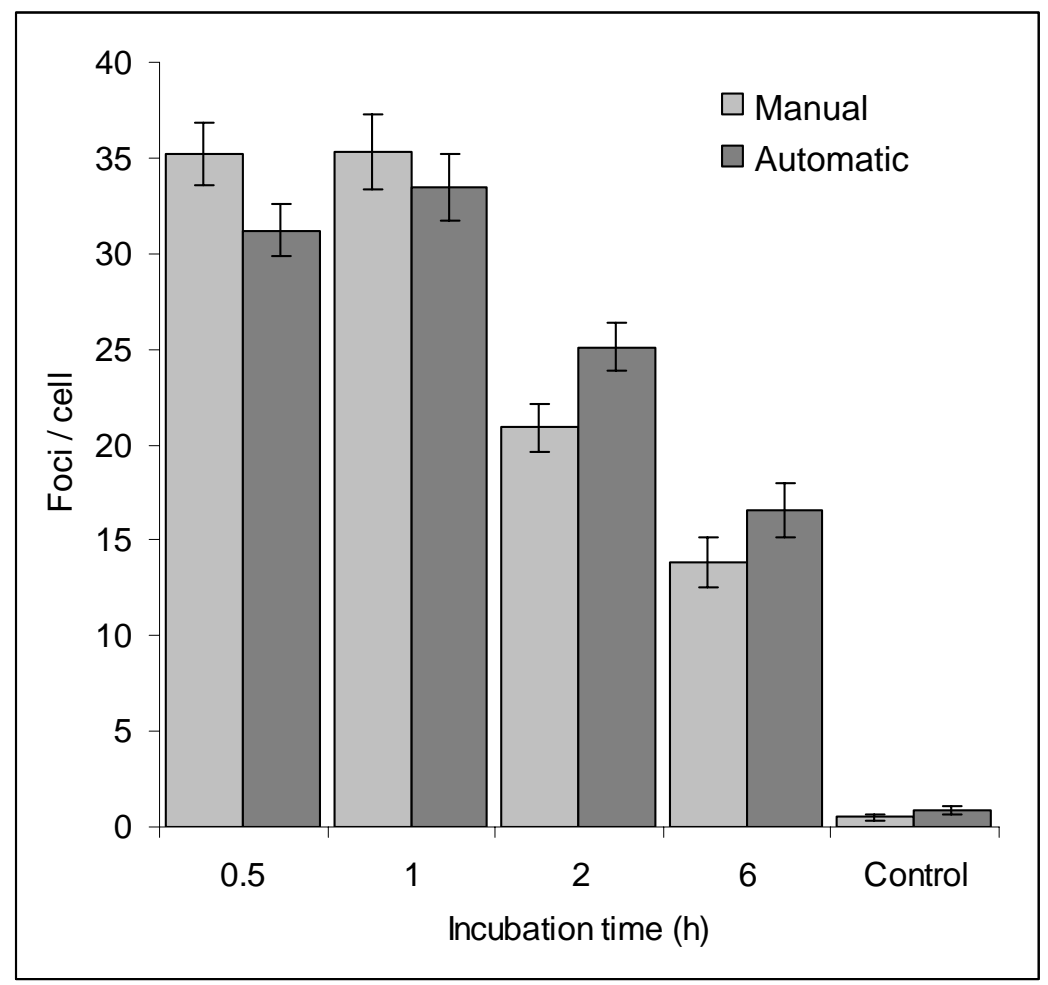

Fig. 6. Results of the initial validation experiment of the time course of foci per cell following irradiation with a 1 Gy X-ray dose. Approximately 50 to 60, 48BRhtert cells were scored per time point. Error bars are standard error in the mean. 


\section{ACKNOWLEDGEMENTS}

The authors gratefully acknowledge the support of Cancer Research UK under programme grants C133/A1812 (P.I.: B. Vojnovic) and C14504/A6116 (P.I.: K. Rothkamm), as well as Francesco Boato for immunofluorescence staining and manual foci scoring.

\section{REFERENCES}

1 Jackson S.P. (2002) Sensing and repairing DNA double-strand breaks. Carcinogenesis 23,687-96.

2 Sedelnikova O.A., Rogakou E.P., Panyutin I.G., Bonner W.M. (2002) Quantitative detection of (125)IdUinduced DNA double-strand breaks with $\gamma \mathrm{H} 2 \mathrm{AX}$ antibody. Radiation Research 158, 486-92.

3 Hamada, N., Schettino, G., Kashino, G., Vaid, M., Suzuki, K., Kodama, S., Vojnovic, B., Folkard, M., Watanabe, M., Michael, B.D. and Prise, K.M. (2006) Histone H2AX Phosphorylation in Normal Human Cells Irradiated with Focused Ultrasoft X Rays: Evidence for Chromatin Movement during Repair. Radiation Research 166, 31-38.

$4 \quad$ Barber, P.R., Vojnovic, B., Kelly, J., Mayes, C.R., Boulton, P., Woodcock, M. and Joiner, M.C. (2001) Automated Counting of Mammalian Cell Colonies. Physics in Medicine and Biology 46, 63-76.

$5 \quad$ Russ, J.C. (1999). The Image Processing Handbook, CRC Press. ISBN 0849325323.

6 Barber, P.R., Locke, R.J., Pierce, G.P., Newman, R.G., Folkard, M. and Vojnovic, B. (2006) The GCI Multitarget X-ray Microprobe: Imaging, Control and Software Automation. Extended Abstracts of the 7th International Workshop: Microbeam Probes of Cellular Radiation Response published in Radiation Research 166, 663-664.

7 Internal GCI technical report: http://www.gci.ac.uk/research/groups/atd/documents/Makeyourownautomatedmicroscope_000.pdf

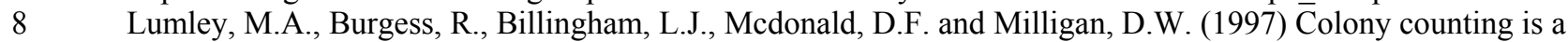
major source of variation in CFU-GM results between centres. British J. Haematology 97, 481-484.

9 Rothkamm, K. and Lobrich, M. (2003) Evidence for a lack of DNA double-strand break repair in human cells exposed to very low x-ray doses. Proceedings of the National Academy of Sciences 100, 5057-5062.

10 Stenerlow, B., Karlsson, K.H., Cooper, B. and Rydberg, B. (2003) Measurement of Prompt DNA DoubleStrand Breaks in Mammalian Cells without Including Heat-Labile Sites: Results for Cells Deficient in Nonhomologous End Joining. Radiation Research 159, 502-510.

11 Goldberg, I.G., Allan, C., Burel, J.M., Creager, D., Falconi, A., Hochheiser, H., Johnston, J., Mellen, J., Sorger, P.K. and Swedlow, J.R. (2005) The Open Microscopy Environment (OME) Data Model and XML file: open tools for informatics and quantitative analysis in biological imaging. Genome-Biol 6, Article R47. http://www.openmicroscopy.org/ 